\title{
Active Frequency Modulation of Metamaterial/Graphene Optoelectronic Device Using Coupled Resonators
}

\author{
S. J. Kindness ${ }^{1} *$, N. W. Almond ${ }^{1}$, R. Wallis ${ }^{1}$, B. Wei ${ }^{1}$, V. S. Kamboj ${ }^{1}$, P. Braeuninger-Weimer ${ }^{2}$, S. \\ Hofmann $^{2}$, H. E. Beere ${ }^{1}$, D. A. Ritchie ${ }^{1}$, and R. Degl'Innocenti ${ }^{3}$ \\ ${ }^{1}$ Cavendish Laboratory, University of Cambridge, J J Thomson Avenue, Cambridge, CB3 OHE, United Kingdom \\ ${ }^{2}$ Dept. of Engineering, University of Cambridge, $9 \mathrm{~J} J$ Thomson Avenue, Cambridge, CB3 OFA, United Kingdom \\ ${ }^{3}$ Dept. of Engineering, Lancaster University, Lancaster, LA1 4YW, United Kingdom \\ *sjk80@cam.ac.uk
}

\begin{abstract}
We present the continuous frequency modulation of a metamaterial resonance using selective damping of coupled plasmonic resonators with electrostatically gated graphene. A resonance frequency tuning range $>150 \mathrm{GHz}$ is achieved at $1.5 \mathrm{THz}$ making this device suitable for use as an optoelectronic, tunable frequency modulator for THz frequencies. (C) 2018 The Author(s)

OCIS codes: (160.3918) Metamaterials; (160.2100) Electro-optical materials; (230.4110) Modulators
\end{abstract}

\section{Introduction}

In recent years there has been a growing interest in using metamaterials to interact with and manipulate $\mathrm{THz}$ radiation. By fabricating metamaterial structures from basic sub-wavelength resonator antennas, the permittivity and permeability of the structure can be lithographically tuned to display optical features not normally observed in nature. In previous works [1,2] metamaterial resonant structures with strong optical reflection at $\mathrm{THz}$ frequencies have been reported which can be variably dampened by the electrostatic gating of monolayer graphene, resulting in amplitude modulator devices as fast as $100 \mathrm{MHz}$. Here, a similar electrical tuning mechanism has been used to demonstrate continuous frequency tuning of a metamaterial resonance whilst maintaining the $\mathrm{Q}$ factor of the resonance, with no detriment to the reconfiguration speed. To achieve this, a metamaterial design based on two strongly coupled resonators has been implemented using graphene's tunable conductivity to variably dampen one of the resonators. By electrically changing the graphene conductivity, the anti-crossing of the resonators can be actively tuned, thus allowing for the resonant peak frequency to be modulated. This high speed optoelectronic frequency manipulation could have applications in multispectral detectors, biochemical sensors, dynamically tunable notch filters and integration with external cavity tuned quantum cascade lasers [3].

\section{Device design and fabrication}

The modulator design is based on two resonators with close resonance frequencies, $\omega_{L}$ (left resonator) and $\omega_{R}$ (right resonator) capacitively coupled together as shown in Fig. 1a). When excited by $\mathrm{THz}$ radiation polarized in the Ey direction, current is induced in the left resonator. Current in the right resonator is mainly excited indirectly from capacitive coupling with the left resonator. Due to this coupling there will be a splitting of the resonance resulting in a low frequency bonding mode at $\omega_{\text {Bonding }}$ and a higher frequency anti-bonding mode at $\omega_{\text {AntiBonding }}$. The device is fabricated on a p -doped silicon substrate $(500 \mu \mathrm{m})$ with a $\mathrm{SiO}_{2}$ insulating layer $(290 \mathrm{~nm})$ on top. E-beam lithography and thermal evaporation of $\mathrm{Ti} / \mathrm{Au}(10-80 \mathrm{~nm})$ is used to fabricate the resonators. Chemical vapor deposition (CVD) grown [4] graphene is then transferred on top of the resonators before being shaped and etched into square areas $\left(5 \times 5 \mu \mathrm{m}^{2}\right)$ using e-beam lithography and oxygen plasma respectively. Fig. 1a) presents a sketch of the metamaterial unit and is illustrating the current directions of the bonding mode present when the resonators are excited with $\mathrm{THz}$ radiation at $\omega_{\text {Bonding }}$.
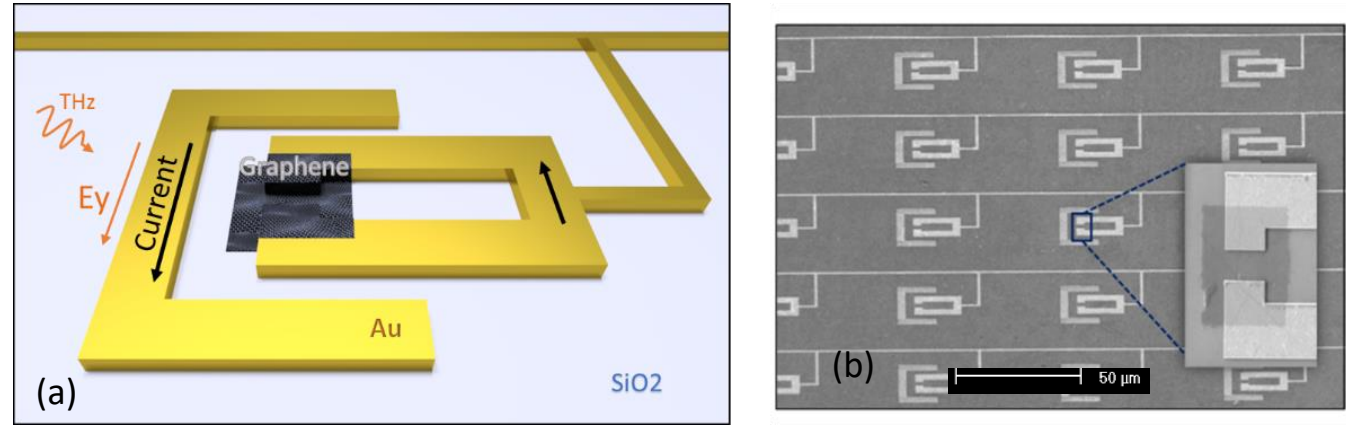

Fig. 1. (a) Sketch of device design illustrating bonding mode of the coupled resonators showing the induced current. (b) SEM image of device array showing gold resonators on top on $\mathrm{Si} / \mathrm{SiO}_{2}$ substrate and dark graphene square shunting the right resonator. 


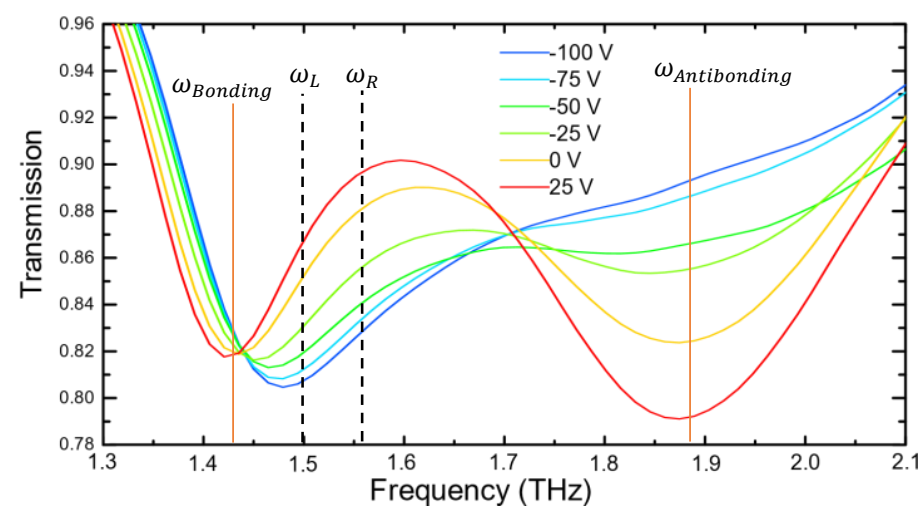

Fig. 2. TDS data of transmission as a function of frequency for different graphene backgate voltages

In order to increase the efficiency of the coupling with external radiation the metamaterial unit is arranged into arrays with a typical size of $1 \times 1 \mathrm{~mm} 2$ (Fig 1b).The horizontal lines shown in Fig.1b) are used to bias the graphene squares and don't interact directly with the incident THz radiation. As the conductivity of the graphene shorting the capacitive gap of the right resonator is increased, the resonator will become more lossy and the individual resonance of the left resonator will then dominate, resulting in a continuous tuning of the resonance frequency from $\omega_{\text {Bonding }}$ to $\omega_{L}$.

\section{Results}

The transmission of the device is measured using a Time domain spectroscopy (TDS) system (TERA K15 from Menlo systems) and normalized to the substrate. As the backgate voltage changes the concentration of free charge carriers in the graphene will change and hence the conductivity can be modulated. A source drain measurement of a separate graphene patch, which was fabricated from the same batch, was performed for different backgate voltages and the Dirac point was measured to be around $25 \mathrm{~V}$ which is consistent with CVD grown p-doped graphene. Fig. 2 shows how the transmission of the device changes as a function of frequency for different backgate voltages. In the metamaterial device, when the graphene is most resistive at $25 \mathrm{~V}$ there are two clear resonances observed at 1.4 and $1.85 \mathrm{THz}$ due to the anti-crossing. As the voltage is decreased from $25 \mathrm{~V}$ to $-100 \mathrm{~V}$ the conductivity of the graphene increases by a factor of 4 allowing us to modulate the strength of the anti-crossing. The two resonances from the resonator coupling transform into one resonance as the right resonator is dampened giving a continuous frequency tuning of $80 \mathrm{GHz}$ between $\omega_{\text {Bonding }}$ and $\omega_{L}$. The amplitude remains relatively constant from $25 \mathrm{~V}$ to $-50 \mathrm{~V}$ but the frequency of the resonance shifts by more than $50 \mathrm{GHz}$. The frequency shift can be increased by lithographically tuning $\omega_{R}$ and $\omega_{L}$ to be closer, in order to increase the interaction strength between the two resonant elements. By doing this, frequency shifts of $150 \mathrm{GHz}$ have been measured in other devices, however the amplitude and Q-factor do not remain as constant, resulting in a less continuous tuning. This electrically tunable coupled resonator design is highly customizable due to the inherent flexibility of the lithographic design of metamaterials, opening up a large range of interesting applications for active dispersion tuning and electromagnetic induced transparency modulation.

\section{Conclusion}

Continuous electronic frequency modulation of a metamaterial resonance is reported at $1.5 \mathrm{THz}$ using coupled resonators and selective graphene damping. This could be used as a fast optoelectronic tunable frequency filter with a tuning range of $80 \mathrm{GHz}$. Due to the flexibility of metamaterials, this device could be scaled to work at any required frequency and could be integrated, for example, with an external cavity quantum cascade laser working in the $\mathrm{THz}$ for dispersion and frequency tuning [3].

\section{References}

[1] Degl'Innocenti, R., Jessop, D. S., Shah, Y., Sibik, J., Zeitler, J. A., Kidambi, P. R., Hofmann, S., Beere, H. B., Ritchie, D. A. "Low-bias terahertz amplitude modulator based on split-ring resonators and graphene." ACS nano 8, 2548-2554 (2014).

[2]Jessop, D. S., Kindness, S. J., Xiao, L., Braeuninger-Weimer, P., Lin, H., Ren, Y., Ren, C. X., Hofmann, S., Zeitler, J.A., Beere, H.E. and Ritchie, D. A., Degl'Innocenti, R. "Graphene based plasmonic terahertz amplitude modulator operating above 100 MHz." Applied Physics Letters 108, 171101 (2016).

[3] Kindness, S. J., Jessop, D. S., Wei, B., Wallis, R. et al. "External amplitude and frequency modulation of a terahertz quantum cascade laser using metamaterial/graphene devices." Scientific Reports 7, 7657 (2017).

[4] Hofmann, S., Braeuninger-Weimer, P. and Weatherup, R.S., 2015. "CVD-enabled graphene manufacture and technology.” The journal of physical chemistry letters 6, 2714-2721 (2015). 